\title{
A 54-year-old man with hallucinations and hearing loss
}

\author{
Claude Steriade MDCM, Steven L. Shumak MD, Anthony Feinstein MD PhD
}

A 54-year-old man who had recently immigrated to Canada presented to the emergency department afraid that "spirits" possessed him. He had long-standing beliefs in a spirit world, rooted in his childhood in Cameroon. In the past few months, he began experiencing command hallucinations from his aunt's spirit, to whom he attributed medical problems, including hearing loss and heart palpitations. The patient had been unemployed for four years and had become socially isolated in the months preceding the presentation. He did not have disorganized behaviour or thought disorder. His mood was not pathologically elevated or depressed. His medical history included atrial flutter (rate controlled with metoprolol) and sudden-onset hearing loss accompanied by transient vertigo and ataxia four years earlier. He had no history of substance abuse and no family history of psychosis.

Clubbed fingers were noted on general medical examination, and bilateral sensorineural hearing loss was found on neurologic examination. Results of laboratory investigations, including a complete blood count, electrolyte levels, and renal and liver function tests, were normal, as was a chest radiograph. The differential diagnosis included delusional disorder, schizophrenia and psychosis secondary to general medical condition.

\section{What differentiates psychosis secondary to general medical condition from schizophrenia?}
a) Later age at onset
b) Preserved affect
c) Lack of thought disorder
d) Multimodal hallucinations
e) All of the above

The correct answer is (e). All of these features are more common in psychosis secondary to general medical condition than in schizophrenia. ${ }^{1,2}$ Our patient presented at a relatively late age and had no personal or family history of psychosis.

A presumed diagnosis of delusional disorder was made, and olanzapine treatment was prescribed on an outpatient basis. Two months later, the patient was brought back to hospital because he was verbally unresponsive, diaphoretic, febrile, tachycardic and hypertensive. His creatine phosphokinase level was markedly elevated (32 935 [normal 60-400] IU/L), and his leukocyte count was normal. Neuroleptic malignant syndrome was diagnosed, and the patient was admitted to the intensive care unit. Because his physical condition improved rapidly with the administration of intravenous fluids and discontinuation of olanzapine, we did not prescribe dantrolene or bromocriptine. Computed tomography $(\mathrm{CT})$ of his brain showed hypodensities in the periventricular white matter, which were thought to be nonspecific and likely representing microangiopathic changes.

After the antipsychotic medication was stopped, the patient's delusions became florid, and he reported being terrified of "spirits" attempting to kill him. Clozapine was started but had to be stopped because neutropenia developed (the neutrophil count dropped from 2.2 to 1.1 [normal $1.5-8.0$ ] $\times 10^{9} / \mathrm{L}$ over three weeks). With pharmacologic options for treating the psychosis exhausted, the patient underwent 12 sessions of electroconvulsive therapy, and his delusions resolved. Although he did not report any subjective cognitive complaints, bedside cognitive testing showed difficulties with executive function, working memory, sustained attention and information processing speed. The neutrophil count remained low after discontinuation of the clozapine (about $1.2 \times 10^{\%} / \mathrm{L}$ ). After the 12 sessions of electroconvulsive therapy, the patient reported right ocular pain and blurred vision. Slit-lamp examination showed right anterior uveitis.
Competing interests: None declared.

This article has been peer reviewed.

The authors have obtained patient consent.

Correspondence to:

Claude Steriade,

claude.steriade@mail .utoronto.ca

CMAJ 2014. DOI:10.1503 /cmaj.131535 


\section{What is your differential diagnosis?}
a) Susac syndrome
b) Limbic encephalitis
c) Mitochondrial disorder
d) Neurosyphilis
e) All of the above

All of the options were worthy of inclusion in the differential diagnosis (e). Susac syndrome, an autoimmune vasculopathy, is characterized by branch retinal artery occlusions, deafness and neuropsychiatric manifestations. Other autoimmune encephalopathies, such as limbic encephalitis, could have explained the patient's neuropsychiatric symptoms. The patient's clubbed fingers may be the result of paraneoplastic limbic encephalitis. His neuropsychiatric symptoms, hearing loss and atrial flutter may be related to mitochondrial disorders. ${ }^{3}$ Neurosyphilis remained a consideration because of the psychosis and uveitis. However, results of a Venereal Disease Research Laboratory (VDRL) test were negative.

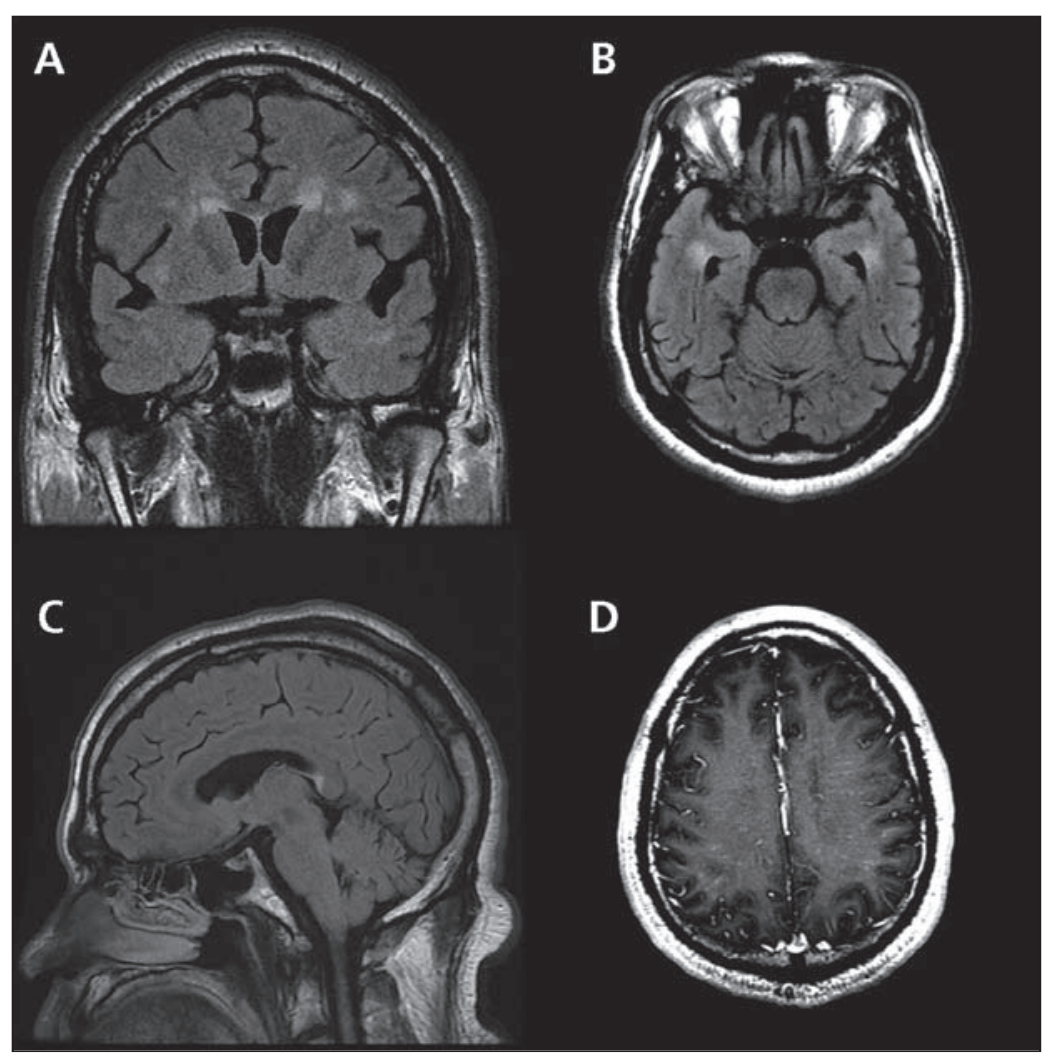

Figure 1: Magnetic resonance imaging of the brain of a 54-year-old man who reported being visited by "spirits." (A) Coronal fluid-attenuated inversionrecovery (FLAIR) sequence, showing symmetric periventricular hyperintensities. (B) Axial FLAIR sequence, showing hyperintensities surrounding the temporal horns. (C) Sagittal FLAIR sequence, showing hyperintensity of the splenium of the corpus callosum. (D): Axial $T_{1}$-weighted image with gadolinium, showing enhancement of the perivascular spaces.
Rheumatologic conditions, such as systemic or primary vasculitis of the central nervous system, can also cause a variety of neuropsychiatric manifestations, and granulomatous processes may seed the leptomeninges, thereby explaining the patient's hearing loss and potentially tying the above signs and symptoms to his uveitis. Inborn errors of metabolism, including metachromatic leukodystrophy, X-linked adrenoleukodystrophy and Niemann-Pick disease type $\mathrm{C}$, have also been known to cause late psychiatric presentations. $^{4}$

The patient's erythrocyte sedimentation rate $(32 \mathrm{~mm} / \mathrm{h})$ and levels of C-reactive protein (4 mg/L), rheumatoid factor $\left(7 \times 10^{3} \mathrm{IU} / \mathrm{L}\right)$, C3 complement ( $1.25 \mathrm{~g} / \mathrm{L})$ and $\mathrm{C} 4$ complement $(0.31 \mathrm{~g} / \mathrm{L})$ were all within normal limits. Tests for antinuclear antibodies and extractable nuclear antigen were negative. His serum angiotensinconverting enzyme (ACE) level was 42 (normal 8-52) U/L. His calcium concentration was 2.28 (normal 2.12-2.57) $\mathrm{mmol} / \mathrm{L}$, with normal levels of albumin and lactate dehydrogenase.

Results of a repeat ophthalmologic evaluation were normal, and we delayed paraneoplastic antibody screening until further brain imaging was performed.

Magnetic resonance imaging (MRI) of the brain with gadolinium showed symmetric hyperintensities of periventricular white matter, including the temporal lobes (Figure 1A and B) and the splenium of the corpus callosum (Figure 1C). Hyperintensities in the temporal lobe are rarely seen in microangiopathic changes and point to inflammatory causes of demyelination. Lesions involving the splenium of the corpus callosum have a broad differential diagnosis, including multiple sclerosis, Susac syndrome, metabolic derangements, neoplasms, alcoholic encephalopathy and rheumatologic diseases. Leptomeningeal enhancement of perivascular spaces (Figure 1D) narrowed the differential diagnosis to rheumatologic diseases such as vasculitis of the central nervous system and granulomatous processes (e.g., sarcoidosis, granulomatous angiitis and lymphomatoid granulomatosis). We placed Susac syndrome lower on the differential diagnosis because of the patient's normal findings on ophthalmologic follow-up examination.

A lumbar puncture showed an erythrocyte count of 6, a leukocyte count of 19 (17 were lymphocytes), protein 0.6 (normal $0.15-0.45) \mathrm{g} / \mathrm{L}$, glucose $3.3 \mathrm{mmol} / \mathrm{L}$, lactate 1.5 (normal $0.5-$ 2.0) $\mathrm{mmol} / \mathrm{L}$ and an ACE level of less than 8 (normal < 8) U/L; there was no oligloclonal banding. No bacteria or fungi were detected in the cerebrospinal fluid, and acid-fast bacilli stain, 
tuberculosis culture and viral polymerase chain reaction yielded negative results. Cytology showed small lymphocytes, but no malignant cells. Flow cytometry revealed a predominant CD3+ T-cell lymphoid cell population.

\section{What is the next diagnostic step?}
a) Leptomeningeal biopsy
b) Electroencephalography
c) Computed tomography of the chest
d) Magnetic resonance imaging of the spine
e) Multimodal evoked potentials testing

Previous investigations had shown a slightly elevated erythrocyte sedimentation rate and negative results of tests for infectious and inflammatory causes. Extensive white matter disease and leptomeningeal enhancement seen on the MRI scans, combined with lymphocytic pleocytosis in the cerebrospinal fluid, suggested neuroinflammation. To detect any underlying systemic condition such as granulomatous disease, vasculitis or neoplasia, we obtained a CT scan of the patient's chest (c). A leptomeningeal biopsy would have been premature given the alternatives available at this stage, but it would be a necessary step if no tissue amenable to biopsy was found systemically. There were no fluctuations of consciousness to suggest seizure; an electroencephalogram, if abnormal, would likely show only nonspecific changes. There were no motor or sensory signs or symptoms to prompt MRI of the spine. Recordings of evoked potentials may indicate subclinical optic nerve disease, which could occur with an infiltrative leptomeningeal process, but would not reveal the cause.

The CT scan of the patient's chest showed enlarged and abnormally numerous internal mammary and axillary lymph nodes and normal lung parenchyma (Figure 2A). The differential diagnosis of the extensive nodal disease included sarcoidosis, lymphoproliferative disorder, metastatic disease and infection. A biopsy of one of the axillary nodes showed non-necrotizing granulomas (Figure 2B). Staining for mycobacteria and fungi yielded normal results, as did polymerase chain reaction for tuberculosis. Probable neurosarcoidosis was diagnosed.

We administered prednisone at an initial dose of $60 \mathrm{mg} / \mathrm{d}$, with no recurrence of the patient's psychosis. After three months, we tapered the dose by $10 \mathrm{mg}$ per month and switched the treatment to methotrexate. At nine-month follow-up, the patient was well from a psychiatric perspective. He was scheduled to receive a cochlear implant. There was no recurrence of uveitis, and no dermal or pulmonary involvement.

\section{Discussion}

Brain involvement occurs in $10 \%$ of patients with sarcoidosis. ${ }^{5}$ Neurosarcoidosis can present with virtually any neurologic symptom, but the most common symptoms include headache, ataxia, cranial nerve palsy (most frequently optic and facial nerves), sensory disturbances in one or more limbs and confusion. ${ }^{6}$ Case series (including 13 to 68 patients) have reported rates of confusion and encephalopathy between $3.4 \%$ and $40 \% .^{6-8}$ This large range may represent a lack of standardized cognitive testing across studies. Psychosis is rarely a presenting symptom but has been reported. ${ }^{9,10}$ Individual patient factors, such as cultural background, may alter the manner in

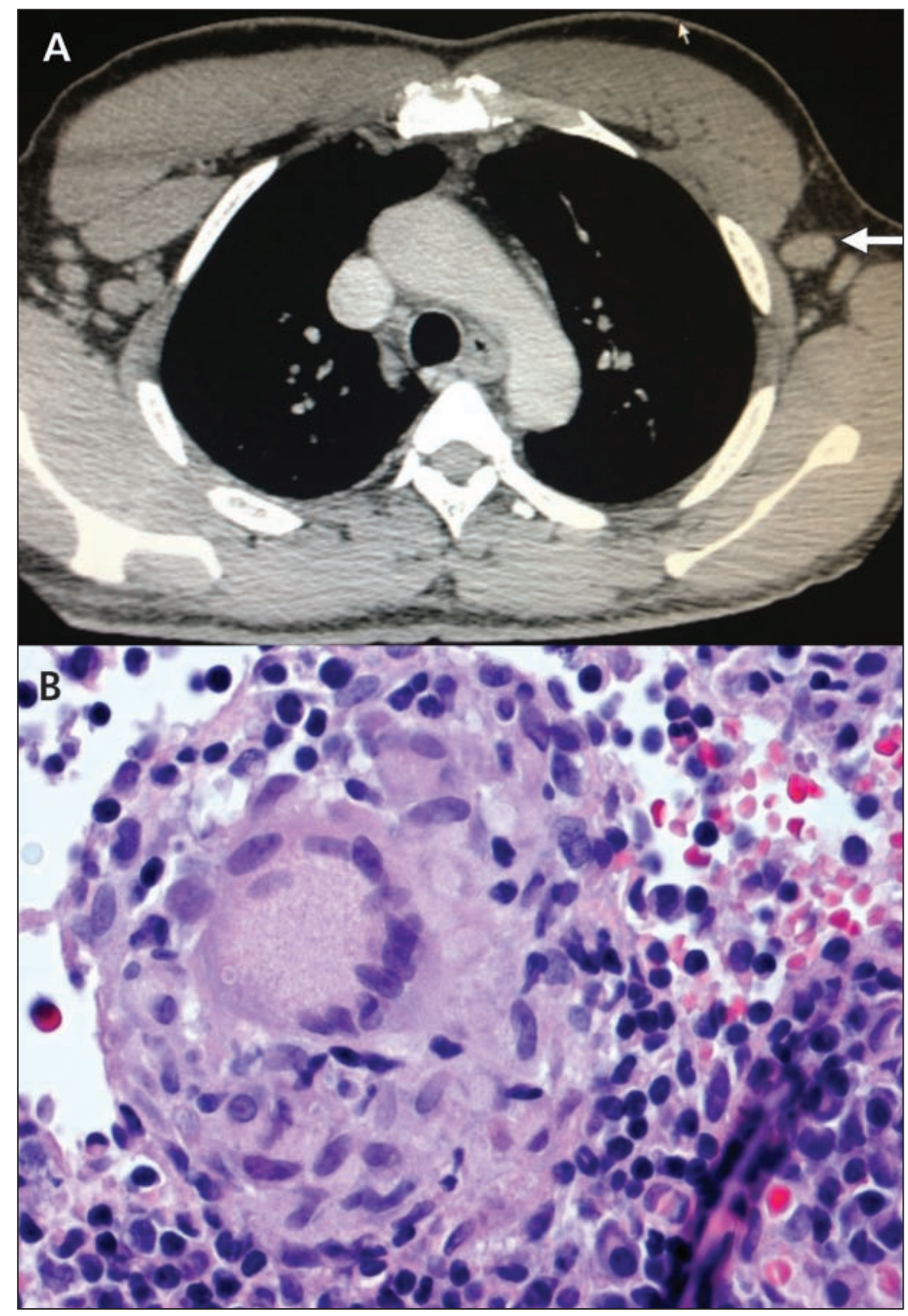

Figure 2: (A) Computed tomography of the patient's chest, showing multiple enlarged lymph nodes; the axillary nodes are prominent (arrow indicates the node that was biopsied). (B) Non-necrotizing caseating granulomas were detected on axillary node biopsy (hematoxylin-eosin, original magnification $\times 100$ ). 
which sarcoidosis manifests neurologically. In our patient's case, the nature of his delusions were consistent with his long-standing beliefs in a spirit world. Vestibulocochlear nerve palsy can occur in $9 \%$ of patients with neurosarcoidosis. ${ }^{11}$ In retrospect, the sudden onset of hearing loss may have been an early indicator of neurosarcoidosis in our patient.

We reached a diagnosis of probable neurosarcoidosis because of a confluence of evidence: indicators of central nervous system inflammation (e.g., clinical and radiographic findings and cerebrospinal fluid measurements) and evidence of systemic sarcoidosis through confirmatory biopsy findings taken from more easily accessible tissue. ${ }^{7}$ Case series support treatment with a high-dose corticosteroid pulse followed by prednisone given orally. ${ }^{5}$ Patients with a poor response to steroids may require more aggressive immunosuppression in the acute phase (e.g., with infliximab ${ }^{12}$ ). One comparative trial showed a slight advantage of steroid-sparing agents over prednisone in the maintenance treatment of neurosarcoidosis. ${ }^{8}$ The natural history of this condition is variable; aseptic meningitis and cranial nerve involvement without brain parenchymal involvement are good prognostic indicators. ${ }^{13}$ Brain parenchymal involvement may be associated with a relapsing-remitting form of disease. ${ }^{13}$

Our patient's case exemplifies the wide clinical presentation associated with neurosarcoidosis. Although his florid delusions initially obscured other systemic signs and symptoms, a stepwise diagnostic approach led to a unifying diagnosis and a good clinical outcome.

\section{References}

1. Feinstein A, Ron M. Psychosis associated with demonstrable brain disease. Psychol Med 1990;20:793-803.

2. Keshavan MS, Kaneko Y. Secondary psychoses: an update. World Psychiatry 2013;12:4-15.
3. Anglin RE, Tarnopolsky MA, Mazurek MF, et al. The psychiatric presentation of mitochondrial disorders in adults. J Neuropsychiatry Clin Neurosci 2012;24:394-409.

4. Walterfang M, Bonnot O, Mocellin R, et al. The neuropsychiatry of inborn errors of metabolism. J Inherit Metab Dis 2013;36:687-702.

5. Iannuzzi MC, Rybicki BA, Teirstein AS. Sarcoidosis. N Engl J Med 2007;357:2153-65.

6. Joseph FG, Scolding NJ. Neurosarcoidosis: a study of 30 new cases. J Neurol Neurosurg Psychiatry 2009;80:297-304.

7. Zajicek JP, Scolding NJ, Foster O, et al. Central nervous system sarcoidosis — diagnosis and management. QJM 1999;92:103-17.

8. Scott TF, Yandora K, Valeri A, et al. Aggressive therapy for neurosarcoidosis: long-term follow-up of 48 treated patients. Arch Neurol 2007;64:691-6.

9. Bona JR, Fackler SM, Fendley MJ, et al. Neurosarcoidosis as a cause of refractory psychosis: a complicated case report. Am J Psychiatry 1998; 155:1106-8.

10. Sabaawi M, Gutierrez-Nunez J, Fragala MR. Neurosarcoidosis presenting as schizophreniform disorder. Int J Psychiatry Med 1992; 22:269-74.

11. Pawate S, Moses H, Sriram S. Presentations and outcomes of neurosarcoidosis: a study of 54 cases. QJM 2009;102:449-60.

12. Santos E, Shaunak S, Renowden S, et al. Treatment of refractory neurosaroidosis with infliximab. J Neurol Neurosurg Psychiatry 2010;81:241-6.

13. Luke RA, Stern BJ, Krumholz A, et al. Neurosarcoidosis: the long-term clinical course. Neurology 1987;37:461-3.

Affiliations: Division of Neurology (Steriade), Department of Medicine (Shumak), Department of Psychiatry (Feinstein), Sunnybrook Health Sciences Centre, University of Toronto, Toronto, Ont.

Contributors: All of the authors contributed to the acquisition and analysis of data. Claude Steriade drafted the manuscript, and Anthony Feinstein and Steven Shumak revised it. All of the authors approved the final version submitted for publication.

CMAJ invites submissions to "What is your call?" Clinical details (including images) are presented with a multiple-choice question about the diagnosis. The answer and a brief discussion of the condition follow. We specifically invite submissions illustrating common or important radiographic and electrocardiographic diagnoses of appeal to a general audience. We require authors to obtain consent from the patient for publication of his or her story (form available at www.cmaj.ca /site/authors/checklist.xhtml). Submit manuscripts online at http://mc.manuscriptcentral.com/cmaj. 